\title{
Unterhaltungsqualität und Public Value
}

von Rimscha, M Bjørn ; De Acevedo, Miriam ; Siegert, Gabriele

\begin{abstract}
Gebühren finanzierte TV Sender haben die Aufgabe Public Value zu schaffen. In der Regel umfasst der Leistungsauftrag dabei sowohl Information und Bildung als auch Unterhaltung. Während sich für informierende und bildende Inhalte relativ leicht feststellen lässt, worin der Nutzen für die Öffentlichkeit besteht, ist dies für die Unterhaltung weniger eindeutig. An dieser Stelle setzt der Beitrag an. Es wird argumentiert, dass der Public Value von Unterhaltung von der Qualität der Unterhaltung abhängt. Hierfür muss zunächst geklärt werden, an welchen Kriterien sich die Qualität von Unterhaltung festmachen lässt. Die Qualität stellt sich vielschichtig dar, es müssen unterschiedliche Perspektiven z.B. von Rezipienten, Produzenten und Regulierern berücksichtigt werden. Auf Basis einer Messung von Qualität wird in einen zweiten Schritt diskutiert, welche Unterhaltungsangebote einen Public Value aufweisen und welchen dieser fehlt, so dass sie nicht zu den Aufgaben eines Service Public Senders zu zählen sind. Dabei zeigt sich, dass eine absolute Bewertung nicht gerechtfertigt ist, sondern jeweils die Kontextbedingungen eines Medienmarkts berücksichtigt werden müssen. Anhand von konkreten Unterhaltungsangeboten von europäischen Service Public Anbietern wird die Messung von Qualität illustriert und mit den Kontextbedingungen in Bezug gesetzt.
\end{abstract}

DOI: https://doi.org/10.1007/978-3-531-92618-6_10

Posted at the Zurich Open Repository and Archive, University of Zurich

ZORA URL: https://doi.org/10.5167/uzh-39712

Book Section

Originally published at:

von Rimscha, M Bjørn; De Acevedo, Miriam; Siegert, Gabriele (2010). Unterhaltungsqualität und Public Value. In: Karmasin, Matthias; Süssenbacher, Daniela; Gonser, Nicole. Public Value. Theorie und Praxis im internationalen Vergleich. Wiesbaden: VS Verlag für Sozialwissenschaften, 141-154.

DOI: https://doi.org/10.1007/978-3-531-92618-6_10 


\title{
Unterhaltungsqualität und Public Value
}

\author{
Bjørn von Rimscha, Miriam de Acevedo \& Gabriele Siegert
}

Die Ansichten darüber, was das Unterhaltungsfernsehen zu Public Value beitragen kann, gehen weit auseinander. Von den Rezipienten wird Unterhaltung genutzt, um in Phantasiewelten einzutauchen, die die Grenzen des Alltags aufheben. Der Zuseher kann sich „Wünsche und Bedürfnisse befriedigen, die ihm die alltägliche Lebenswelt mit ihren spezifischen Relevanzbereichen versagt" (Stumm/Dorsch-Jungsberger 1996: 132). Je nach Weltanschauung kann diese Entspannung nun als funktional und gesellschaftlich gewünscht oder als dysfunktional und gesellschaftsschädlich angesehen werden. Die Diskussion um den Wert von Unterhaltung ist so alt wie das öffentliche Fernsehen. Lange bevor der Begriff Public Value als Beschreibung der eigenen Leistung in das Vokabular von Verantwortlichen bei Public-Service-Sendern aufgenommen wurde (vgl. Freedman 2008) wurde gestritten, was der Beitrag der Unterhaltung zum Auftrag der öffentlichen Sender sein könnte. So haben sich z. B. die Mainzer Tage der Fernsehkritik 1970 der Unterhaltung im Fernsehen gewidmet. Der damalige Geschäftsführer der Bavaria Studios propagierte eine paternalistische Meritorik (vgl. Erlei 1992) wenn er erklärt, es sei die Aufgabe der Programmverantwortlichen, das Publikum ,daran zu hindern, das zu wollen, was seinem Nutzen, seiner Freiheit und seiner Orientierung in die Zukunft entgegensteht" (Gottschalk 1971: 109). Scheuch (1971) dagegen glaubt nicht, dass der öffentliche Rundfunk durch ein erzieherisch gemeintes Unterhaltungsprogramm die Bedürfnisse des Publikums lenken könne. ${ }^{1}$ Entsprechend wundert er sich über damals unter Medienschaffenden populäre Forderungen, wonach der öffentliche Rundfunk sich als Anstalt zu Beförderung von Umstürzen verstehen sollte, indem die Rezipienten der Berieselung entrissen werden sollten.

Mit Auftreten der kommerziellen Sender in den 1980er Jahren hat sich die Diskussion verändert. Das kommerzielle Ziel der neuen Sender lässt es sinnvoll erscheinen, die Unterhaltung so zu gestalten, dass sie niemanden ausschließt

1 Gleichwohl wurde dies versucht: Hans Jürgen Weiss erinnert sich an den Versuch mit „volkspädagogischer Intention“ das Publikum an Information heranzuführen indem am Donnerstag das Programm weitgehend unterhaltungsfrei war (in Metz und Seeßlen 2009). Gleichzeitig wurden zwischen der ARD und dem ZDF abgesprochen z. B. politische Magazine parallel zu senden um den Unterhaltungssuchenden Zuschauern die Alternative zu nehmen (Bleicher 2001: 503). 
und mit einfachen Mitteln emotionale Primärtriebe (z. B. Sexualität und Entspannung) befriedigt. Dem Angebot der kommerziellen Sender wird ein Public Value weitestgehend abgesprochen, vielmehr würden sie zur Verrohung der Gesellschaft und zur Verblödung der Jugend beitragen (vgl. z. B. Kissler 2009). Daneben läuft die Debatte weiter, inwieweit auch „Marienhof“ und der „Musikantenstadel" zum öffentlichen Auftrag gehören. Unterhaltung in öffentlichen Sendern bezieht mittlerweile seinen Public Value häufig aus dem Kontrast: Das Angebot gilt als wertvoller, weil bestimmte Themen oder Inszenierungen eben nicht gebracht werden (vgl. z. B. Amgarten 2008). Immer noch ist Public Value jedoch ein zutiefst normativer Begriff, wie Niggemeier mit seiner sarkastischen Definition deutlich macht: „Public Value ist, wenn das läuft, was ich gerne sehen will" (Niggemeier 2008: 156). Hier wird Gemeinwohl und individueller Geschmack gleichgesetzt. Das Problem bleibt jedoch, dass sich für informierende und bildende Inhalte leichter als für Unterhaltung feststellen lässt, worin der Nutzen für die Öffentlichkeit besteht. Wenn Public Value als Gemeinwohl oder Meritorik beschrieben wird, müssen diese Begriffe für die Unterhaltung mit Leben gefüllt werden. Gemeinwohl kann, gleichsam als Nebenprodukt, auch bei der Produktion von „Private Value“ im kommerziellen TV entstehen. Meritorische Güter werden von kommerziellen Anbietern dagegen per definitionem nicht ausreichend bereitgestellt. Für diesen Beitrag werden wir die letztere Perspektive einnehmen. Nicht alle Sendungen, die der Public Service anbietet, haben meritorischen Charakter. Während sich in den 1970er die Diskussion jedoch um die Frage drehte, was denn das Fernsehvolk wollen sollte, kann nach der Zulassung der kommerziellen Anbieter die Ideologie ein wenig aus der Diskussion herausgenommen werden. Die schlichte Frage lautet: Welche Unterhaltungsangebote werden von den kommerziellen Anbietern nicht bereitgestellt, sind aber aus gesamtgesellschaftlicher Perspektive wünschenswert? Es geht also nicht darum, welche Unterhaltung per se "gut“ ist, sondern welche nur dank Gebühren entstehen kann. ${ }^{2}$ Lobigs (2004) argumentiert, dass niveauvolle Unterhaltung die Voraussetzung für ein Argument der Meritorik sei. Meritorik und damit Public Value werden somit an einer hohen Qualität festgemacht. In Kleinstaaten wie Österreich oder der Schweiz ist Qualität jedoch nicht der einzige Aspekt von Public Value. Hier geht es auch um die Konstruktion und Pflege von nationaler Identität. So bekommt selbst eine Sendung wie der Musikantenstadl seine Relevanz und Rechtfertigung jenseits der Gebührenakzeptanz (vgl. Binder/Fartacek 2006a). Gleichzeitig wird in kleinen Märkten der PublicService-Anbieter häufig zu einem erheblichen Anteil auch aus Werbeeinnahmen Meritorik nicht die einzige Begründung für den Public Service sieht. Eine weitere ist z. B. auch die erwünschte organisationale Vielfalt. 
finanziert, zum einen, weil bei der vergleichsweise geringen Zahl von Gebührenzahler andernfalls die Gebühr prohibitiv hoch sein müsste und zum anderen, weil die Sender in der Regel die wichtigste audiovisuelle Werbeplattform in dem Land darstellen, auf das die Werbung treibende Wirtschaft nicht verzichten will. Somit kann festgehalten werden, dass Public Service Sender in Kleinstaaten in ihrer Werbeabhängigkeit ökonomisch kommerziellen Anbietern ähnlicher sind. Public Value wird eher an der Integrationsleistung als an der Orientierung an Minderheiten oder Eliten festgemacht. Es zeigt sich, dass die Gleichsetzung von Public Value mit Qualität voraussetzt, dass klargestellt wird, was Qualität ausmacht. Im folgenden Abschnitt wollen wir deshalb verschiedene Perspektiven der Qualität von TV-Unterhaltungssendungen beleuchten und aufzeigen, dass nicht alle Aspekte der Qualität gleichermaßen relevant für den Public Value sind.

\section{Perspektiven auf Qualität}

Die Forschung zu den Nutzenmotiven legt nahe, dass unterhaltende Inhalte vor allem zur Befriedigung von Bedürfnissen in den Bereichen Ästhetik (Harmonie, Schönheit etc.), Psyche (Ablenkung, Flucht, Stabilität, Entspannung, Freude, aber auch Stimulation und Abwechslung etc.) und Sozialpsyche (Orientierung, Integration, Anerkennung) rezipiert werden (vgl. z. B. Bosshart 2006).

- Aus Rezipientenperspektive könnte man solchen Sendungen Qualität zusprechen, welche die genannten Bedürfnisse befriedigen. Dies würde jedoch bedeuten, Qualität weitgehend mit Akzeptanz gleichzusetzen. Wober (1990) stellt dagegen fest, dass das Publikum unterschiedliche Maßstäbe zu Bewertung von Qualität verwendet: Qualitätsurteile lassen sich von Gefallensurteilen unterscheiden und sind deutlich strenger. Qualität wird erkannt ${ }^{3}$ und ist sozial erwünscht, wird aber nicht notwendigerweise nachgefragt. Nach einem eindeutigen Indikator für Qualitätsfernsehen befragt, sagen 27 Prozent der Befragten, ein Programm muss kurzweilig und unterhaltsam sein, nur zwölf Prozent nennen Eigenschaften wie informativ oder aufklärend. Informationsprogramme werden stärker mit Qualität assoziiert als Fernsehfilme, diese wiederum stärker als Unterhaltungsshows. Costera Meijer (2005: 37) schließt daraus, dass Qualität als Genrecharakteristikum von ernsthaften Sendungen verstanden wird und nicht als neutrale Eigenschaft, die das Publikum animiert, eine bestimmte Sendung zu sehen. Aber auch die Unterhaltung zum Spaß und Zeitvertreib hat eine soziale Funktion und damit einen

3 Das Erkennen wird dabei häufig nicht an einzelnen Programmeigenschaften, sondern an der Gattung festgemacht. Auf Österreich übertragen hieße das z. B., die Zuseher erkennen Dieter Mohr bei „les.art“, halten das Programm ggf. wegen seiner Aufmachung für Qualität, und dennoch schaltet ein Großteil um. 
Qualität im Sinne des Public Value. Bereits Friedrich Schiller beschrieb die integrierende Leistung der Kunst durch das Teilen von gemeinsam empfundener Freude (vgl. Shusterman 2003: 304).

- Die Kommunikatorperspektive ist weniger untersucht als die Rezipientensicht. In Hand- und Lehrbüchern für die Ausbildung kommt Qualität allenfalls als Signalqualität vor. In den 1990er Jahren haben Nossiter (1991), Blumler (1991), Albers (1992) und Leggatt (1993) jeweils qualitative Interviews mit Produzenten durchgeführt um ihre Arbeitsdefinition von Qualität zu erfassen. Ihre Ergebnisse zeigen, dass Qualität ein relativer Begriff ist, der sich einer eindeutigen Definition entzieht und stattdessen eher als diffuses, aber positiv konnotiertes Ideal dient. Anhaltspunkte finden sich in der technischen Umsetzung, in Produzenteneigenschaften wie z. B. Leidenschaft, in Programmeigenschaften wie z. B. Ernsthaftigkeit und Relevanz, in Innovation, in Ressourcen und schließlich in der Zuschauerreaktion. Insbesondere Produzenten- und Programmeigenschaften sind dabei stark normativ beeinflusst: Relevanz z. B. will definiert sein, und der Wert von Leidenschaft ändert sich ggf. mit ihrem Objekt. Aus Kommunikatorperspektive ist Qualität somit subjektiv: Ziel ist es, eben nicht das naheliegendste und billigste Angebot zu produzieren. Die Erwartung der Gremien von öffentlichen Sendern ist denn auch ein persönliches Engagement der Kommunikatoren für Qualität:

„Hier darf Qualität nicht nur eine Frage des Geldes und des Gehaltes sein. Hier muß, jedenfalls nach meiner Überzeugung, etwas hinzutreten, was mit anerkannter Verpflichtung zu tun hat, dem Gemeinwohl zu nutzen. “ (ZDF-Fernsehrat Klär 1995)

- Aus Distributorperspektive lässt sich Qualität an der Frage messen, wie gut ein Programm geeignet ist, den Unternehmenszwecken zu genügen. Im Falle von kommerziellen Anbietern bedeutet dies ein gutes Verhältnis zwischen den Beschaffungskosten einer Sendung und den Werbeumsätzen, die mit ihr erzielt werden. Auch Public-Service-Anbieter müssen die Kosten kontrollieren; vor der Quote sollte aber noch die Erfüllung der im Programmauftrag festgelegten gesellschaftlichen und kulturellen Anforderungen stehen. Controlling für Public-Service-Sender beinhaltet deshalb notwendigerweise auch eine Qualitätskomponente. Das Schweizer Fernsehen hat hier eine Vorreiterrolle übernommen (Schlote/Latzel 1998; Wildberger 1994). Bereits 1993 wurde eine Sendungserfolgskontrolle mit drei quantifizierbaren Kriterien eingeführt: (1) Kosten der Sendung, (2) Marktanteil der Sendung und (3) Reputation der Sendung. In letzteres Kriterium fließen sowohl die Akzeptanz beim Publikum, als auch die Resonanz in anderen Medien und der Öffentlichkeit, nicht jedoch die normativ verstandene Relevanz des 
Themas ein. Auch bei einem Public-Service-Sender scheint somit das Minimalprinzip zu gelten: Bei minimalen Kosten eine ausreichende Qualität erreichen.

- Die Perspektive der Kritiker wird in denjenigen Kriterien deutlich, die bei der Vergabe von Auszeichnungen für einzelne Sendungen angewendet werden. Bei Preisen, die von Peers vergeben werden, spielen vor allem implizite Berufsnormen eine Rolle, aber auch sachfremde wie Sympathie. Bei Preisen, die klare Kriterien anwenden, dominieren formale Kriterien über inhaltliche (Albers 1996). Insgesamt finden sich in der Kritikerperspektive viele subjektive Werturteile mit eigenem Gestaltungswillen. Janke (1980) forderte etwa Fernsehkritik müsse politische Kritik sein.

- In der Reguliererperspektive muss die von Gesetz und Gesellschaft vorgegebene Verpflichtung zu Qualität messbar gemacht werden und das Vorhandensein respektive Fehlen zu einem justiziablen Tatbestand gemacht werden. Somit sollte man in der Reguliererperspektive die eindeutigste Definition finden können, welche Eigenschaften Qualität ausmachen. Das Gegenteil ist der Fall. Ein Blick in die relevanten Rechtstexte für Public-Service-Sender in Europa zeigt, dass das Problem der Qualität entweder ausgeblendet oder als unbestimmter Rechtsbegriff nur vage umrissen wird (vgl. den folgenden Abschnitt). Eine klare Definition von Qualität könnte als Zensur missverstanden werden und damit Kunst- und Meinungsfreiheit widersprechen. Es lassen sich zwei Strategien beobachten, wie mit diesem Widerspruch umgegangen wird: Entweder wird Qualität auf der Metaebene adressiert, indem Veranstalter zur Einrichtung eines Qualitätssicherungssystems und zum entsprechenden Reporting verpflichtet werden. Alternativ werden im Programmauftrag nicht abschließende Listen aufgeführt, welche Inhalte und Produktionsformen mindestens vorkommen müssen, und es wird festgehalten, dass sich ganz allgemein das Angebot von Public Service Sendern von der kommerziellen Konkurrenz unterscheiden müsse.

Public Value in der TV-Unterhaltung ergibt sich aus der Qualität, die das Angebot aufweist, wobei Qualität hier insbesondere aus Perspektive der Regulierung verstanden werden muss. Alle anderen Perspektiven auf die Qualität bilden Partikularinteressen ab, die jeweils einen speziellen privaten Value höher gewichten als das Gemeinwohl. Doch auch aus Reguliererperspektive ergibt sich keine eindeutige Definition. Einen erheblichen Einfluss haben die Kontextbedingungen des jeweiligen Medienmarkts, die dazu führen können, dass z. B. entweder Integration oder Vielfalt stärker gewichtet werden. Im folgenden Abschnitt wird insbesondere am Beispiel von Kleinstaaten aufgezeigt, welchen Einfluss die Marktbedingungen auf das Verständnis von Qualität und damit Public Value haben. 


\section{Kontextbedingungen der Märkte}

Regulierungskonzepte variieren von Land zu Land: Je nach gesellschaftlicher Entwicklungsphase und Größe des Staates kommt es zu bestimmten Regelungsformen für Rundfunkveranstalter. Grundsätzlich tendieren Kleinstaaten zu interventionistischer und protektionistischer Regulierung (Puppis 2009: 14). In Kleinstaaten mit gleichsprachigen Nachbarländern wie z. B. der Schweiz oder Österreich ist die Rundfunklandschaft durch eine hohe Präsenz ausländischer Programme und eine starke Auslandsorientierung des Publikums geprägt. Für TV-Sender in Kleinstaaten ist es daher schwieriger, kulturelle und soziale Leistungen für die Gesellschaft zu erbringen. Durch staatliche Regulierung und eine herausragende Rolle des Public Service wird versucht, die kulturelle Identität des Landes zu wahren, um sich durch die Stärkung der eigenen Kultur vom großen Nachbarn abzugrenzen. ,A small country can only viable affirm its audiovisual identity and produce quality programming by defending the role of public service“ (Burgelman/Pauwels 1992: 174). Doch auch in einigen großen Ländern finden sich Tendenzen, mehr Vorgaben über den Inhalt und die Qualität zu machen. Frankreich sieht sich gegenüber der angelsächsischen Welt häufig in der Defensive, und entsprechend wird in der Regulierung ein Beitrag der TV-Sender zur Pflege der Französischen Sprache und Kultur verlangt. Auf eine weitreichende Selbstkontrolle der Public-Service-Veranstalter vertrauen demgegenüber die deutsche und britische Regulierung (Holznagel/Vollmeier 2003: 278). Die Medienstruktur eines Landes ist mit den Regulierungskonzepten und den damit verbundenen Absichten und Zielen für gebührenfinanzierte TVSender wechselseitig verknüpft. Die vom Gesetzgeber formulierten Rahmenbedingungen müssen von den Public-Service-Sendern eingehalten werden. Aber auch private Veranstalter unterliegen einer gesetzlichen Regulierung. PublicService-Veranstalter dürfen vom Gesetzgeber jedoch nur als staatsunabhängige Organisationen eingerichtet werden. Die Autonomie der Medienschaffenden in der Berichterstattung muss berücksichtigt und gesichert werden (HoffmannRiem 2003: 32). Neben den vom Gesetzgeber geschaffenen oder sich aus der Konzession ergebenen Vorgaben für das Handeln von Akteuren der Public Service und kommerziellen Sender bestehen innerhalb der jeweiligen Organisation weitere Dokumente, welche die normativen Vorstellungen und Zielsetzungen der institutionalisierten Regulierungskonzepte in interne Rahmenbedingen übersetzen. Dokumente, wie beispielsweise ein Leitbild, sind nicht ausschließlich auf der normativen Ebene angesiedelt, sondern in der Mittelposition ,zwischen normenvermitteltem Wert und realer Erfahrung“ (Jarren 1996: 74). Diese Dokumente dienen im Sinne einer Selbstverpflichtung als zusätzliche Rahmenbedingung, die ein Veranstalter seinen Mitarbeitenden vorgibt. 
In den externen rechtlichen Rahmenbedingungen, welche durch den Gesetzgeber formuliert werden, und in den internen Rahmenbedingungen sind konkrete Vorgaben zur Qualität der Programme Mangelware. Der Qualitätsbegriff wird zwar verwendet, es wird jedoch nicht darauf eingegangen, was Qualität explizit ist und wie Qualität durch die Veranstalter gesichert werden soll. Auch lassen sich keine großen Unterschiede zwischen Public-Service- und kommerziellen Sendern diesbezüglich aufzeigen. Vielmehr sind in den Dokumenten Gemeinsamkeiten darzustellen: Die Trennung von Werbung und Programm, die Einhaltung des Jugendschutzes, der Grundsatz der Meinungsfreiheit, die Sicherung von Meinungsvielfalt und die Erfüllung der Programmquoten sind Verpflichtungen, welche für die Public-Service- und kommerziellen Sender gleichermaßen gelten. Für die Public-Service-Sender gilt, dass sie sich von den kommerziellen Anbietern differenzieren sollen. Hier dominieren in den Gesetzen und Verordnungen unbestimmte Rechtsbegriffe, welche vom Public-ServiceFernsehen mehr „Anspruch“, ein höheres „Niveau“, mehr Anstrengungen für die Integration der Gesellschaft und für die Kooperation mit der audiovisuellen Branche verlangen. Für die beiden Kleinstaaten Schweiz und Österreich lassen sich konkretere Angaben zur Qualitätssicherung aufzeigen. Im Bereich der Qualität geht die Schweizer Regulierung über den in den Nachbarländern üblichen Rahmen hinaus. Mit der Revision des Radio- und Fernsehgesetzes (RTVG), der Radio- und Fernsehverordnung (RTVV) und der Erneuerung der Konzessionen für die Veranstalter wurde eine Positivkontrolle der Programmqualität eingeführt. Es soll nicht mehr nur nachträglich bei Verstößen gerügt und die Programmaufsicht verdachtsunabhängig institutionalisiert werden. Qualitätsstandards sind in der Konzession benannt und müssen von der Schweizer Rundfunkgesellschaft SRG selbst konkret definiert und veröffentlicht werden. Die Einhaltung dieser Standards wird anhand von externen und internen Programmanalysen und Publikumsbefragungen überprüft. Die SRG ist insofern in Europa ein Einzelfall, als durch die Konzession definiert wird, was unter Qualität zu verstehen ist. Die Programme müssen sich durch „Glaubwürdigkeit, Verantwortungsbewusstsein, Relevanz und journalistische Professionalität" (Konzession SRG, Art. 3) auszeichnen. Österreich, als weiterer Kleinstaat, ist intern zwar homogener als die Schweiz, doch auch hier gibt es neben der eigenständigen Kultur einen gleichsprachigen großen Nachbarn, dessen Medien von der Bevölkerung stark genutzt werden. Dies bedeutet auch hier eine gewisse Verletzlichkeit des kleinstaatlichen Kommunikationsraums und die Gefahr einer ökonomischen Abhängigkeit. Anders als in vielen anderen Rundfunkgesetzen im europäischen Raum erwähnt das Bundesgesetz über den Österreichischen Rundfunk (ORF-G) explizit die gesellschaftliche Relevanz der Unterhaltung und fordert ein entsprechendes Verantwortungsbewusstsein ein: „Die Unterhaltung soll 
nicht nur die unterschiedlichen Ansprüche berücksichtigen, sondern auch den Umstand, dass sie wie kaum ein anderer Bereich Verhaltensweisen, Selbstverständnis und Identität prägt" (ORF-G, § 10, Abs. 10). Eine Besonderheit des ORF-G ist der explizite Bezug auf die Mitarbeitenden des Senders. In $\S 4$ Abs. 7 ORF-G heißt es: „Die Mitarbeiter des Österreichischen Rundfunks sind den Zielen des Programmauftrags verpflichtet und haben an dessen Erfüllung aktiv mitzuwirken." Insofern sollten ORF Mitarbeitende stärker als andere mit den externen Rahmenbedingungen für ihre Arbeit vertraut sein.

Zusammenfassend lässt sich konstatieren, dass vor allem bei den PublicService-Sendern aus Kleinstaaten mehr durch den Staat eingegriffen wird, um so vorwiegend die eigene Kultur zu wahren. Durch die strikteren Angaben, vor allem für die Schweizer Public-Service-Sender in Bezug auf Qualität, wird so ein Beitrag zur Schaffung und Sicherung von Public Value geleistet.

Wenn wir nun die vier Qualitätsdimensionen aus der Konzession SRG zur Grundlage nehmen, können wir an Beispielen aufzeigen, welchen Sendungen ein Public Value innewohnt und welchen eher nicht. Leser, die hier auf eine universelle Public-Value-Skala gehofft haben, müssen enttäuscht werden. Public Value verstanden als meritorische Qualität bleibt auch mit den Dimensionen Glaubwürdigkeit, Verantwortungsbewusstsein, Relevanz und Professionalität ein relativer Begriff, der von den jeweiligen Marktbedingungen abhängig ist. Somit kann dasselbe Sendekonzept z. B. in einem Kleinstaat einen meritorischen Public Value aufweisen, in einem größeren Land dagegen kann es ggf. problemlos vom Markt bereitgestellt werden.

\section{Wer hat Public Value? Fallbeispiele}

Anhand von konkreten Unterhaltungsangeboten von europäischen PublicService-Anbietern wird die Messung von Qualität illustriert und mit den Kontextbedingungen in Verbindung gesetzt. „SF bi de Lüt - Über Stock und Stein“ (SF1) soll als Beispiel für eine qualitativ hochstehende Sendung mit klarem Public Value beleuchtet werden. ,alfredissimo“ (ARD) hingegen ist Beispiel für ein Unterhaltungsangebot in der Grauzone am Rande des Public Value, und schließlich soll der Sport als ein Bereich beschrieben werden, in dem Unterhaltungsangeboten gelegentlich kein Public Value attestiert werden kann.

„SF bi de Lüt - Über Stock und Stein“ ist eine vom Schweizer Fernsehen eigenproduzierte Sendung, welche jeweils freitags um 20.05 Uhr auf SF1 ausgestrahlt wurde. „SF bi de Lüt" ist keine einzelne Sendung, sondern ein Label, unter dem die Sendungen der Redaktion Volkskultur ausgestrahlt werden. In diesem Rahmen zeigte das SF „Über Stock und Stein“ und begleitete den Moderator und seine Hündin in mehreren Etappen quer durch 10 Kantone der Schweiz. Das Ziel der Sendung war es, den Zuschauerinnen und Zuschauern die 
Schönheiten und Eigenheiten der Schweiz auf positive und lustvolle Weise zu präsentieren und die Fernsehzuschauerinnen und -zuschauer rechtzeitig zum Start der Wandersaison zum Mit- und Nachwandern anzuregen. „Über Stock und Stein" kann aus mehreren Gründen als Sendung mit Public Value bewertet werden: Bezogen auf die Kleinstaatlichkeit der Schweiz trägt die Reisesendung „SF bi de Lüt - Über Stock und Stein“ zur kulturellen Selbstversicherung der Schweiz bei. Die Schweizer Bevölkerung wird anhand der Sendung durch die schönsten Regionen der Schweiz geführt und lernt noch unbekannte Orte der Schweiz kennen. Die kulturelle Selbstversicherung gehört zum Leistungsauftrag des Schweizer Fernsehens. So wird in der Bundesverfassung thematisiert, dass die Public-Service-Sender die Besonderheiten des Landes und die Bedürfnisse der Kantone berücksichtigen sollen (BV, Art. 93). „SF bi de Lüt - Über Stock und Stein“ erfüllt durch die Bekanntmachung der Schweizer Regionen diesen Auftrag, und Qualität in diesem Sinne ist gewährleistet. Qualität wird ferner dadurch gesichert, dass die Sendung eine Eigenproduktion darstellt. Bei Eigenproduktionen kann stärker auf qualitativen Anforderungen geachtet und leichter einen Anpassung an die Schweizer Kultur realisiert werden. Angelehnt an Schatz und Schulz (1992) werden die Kriterien Professionalität und Relevanz durch diese Sendung erfüllt. Bezogen auf Qualitätsbewertung in Zusammenhang mit der Rezipienten- und Regulierungsperspektive nach Gehrau (2008) erfüllt diese Unterhaltungssendung auch diese Qualitätskriterien. Aus der Rezipientenperspektive werden die Kriterien Verantwortungsbewusstsein und Glaubwürdigkeit durch den TV-Sender eingehalten. Qualität wird somit durch den Aspekt des Jugendschutzes gewährleistet, denn die Sendung ist sowohl für Erwachsene als auch für Jugendliche konzipiert. Glaubwürdigkeit wird der Sendung dadurch verliehen, dass die Sendung zum Wandern anregen soll und der Moderator selbst durch die Schweizer Regionen wandert. Die Routen und ein Reisetagebuch können unter anderem auf der Webseite gesichtet werden. Aus der Regulierungsperspektive werden die vier in der Konzession formulierten Qualitätskriterien Glaubwürdigkeit, Verantwortungsbewusstsein, Relevanz und journalistische Professionalität eingehalten. „SF bi de Lüt - Über Stock und Stein“ kann somit als Beispiel für eine Unterhaltungssendung, welche Public Value bietet, bestimmt werden. 
„alfredissimo“ ist dagegen ein Beispiel für eine Unterhaltungssendung, deren Public Value weniger klar zu bestimmen ist. Die Sendung wurde von 1994 bis 2006 im Auftrag des WDR produziert und von Alfred Biolek moderiert. ${ }^{4}$ Produziert wird die Sendung von der Pro GmbH, welche von Alfred Biolek und Andreas Lichter gegründet wurde. Bei „alfredissimo“ wird jeweils ein prominenter Gast in die vorgeblich private Küche eingeladen, um gemeinsam mit Alfred Biolek vor der Kamera zu kochen. Als Sendung, die das Kochen zum Thema hat und kochfreudige Prominente präsentiert, gibt sie dem selber Kochen im Kontrast zum Convenience Food einen gesellschaftlichen Stellenwert, und so kann argumentiert werden, dass ,alfredissimo“ Public Value bietet. „alfredissimo“ leistet weiter Public Value insofern, als sich die Sendung von anderen Kochsendungen differenziert, da sie nicht kommerziell konzipiert ist: Dadurch dass ,alfredissimo" komplett vom WDR finanziert wird und somit nicht auf weiteres Sponsoring angewiesen ist, werden in der Sendung keine Markenprodukte präsentiert, welche von den Rezipienten bestenfalls gekauft werden sollen. Bei einer Sendung, welche jedoch durch die Produktionsfirma des Moderators produziert wurde, könnte aus Rezipientenperspektive von den Zuschauern grundsätzlich ein Interessenkonflikt unterstellt werden. Die Redaktion leitet auch aus dem Public-Value-Auftrag keine Verpflichtung zur Promotion von gesunder oder umweltfreundlicher Ernährung ab. Vielmehr ist das Markenzeichen der Sendung die Weinprobe, was allerdings aus Perspektive des Jugendschutzes oder der Suchtprävention nicht unproblematisch ist. Legt man den Maßstab der Substituierbarkeit (Dewenter/Haucap 2009) zugrunde, muss ,alfredissimo“ der Public Value abgesprochen werden. Die Vielzahl der Kochsendungen auf kommerziellen TV-Sendern im deutschsprachigen Raum belegt, dass keine Marktunvollkommenheit vorliegt, die durch ein Public-Service-Angebot ausgeglichen werden müsste. Dies gilt umso mehr, da „alfredissimo“ kaum qualitativer Mehrwert gegenüber der kommerziellen Konkurrenz attestiert werden kann.

Beispiele für Sendungen im Public Service, denen ein Public Value komplett fehlt, lassen sich insbesondere im Kontext von sportlichen Großereignissen ausmachen - dort trifft die Kommerzialisierung des Sports auf das Trennungsgebot im Public Service. Im Vorfeld der Olympischen Spiele in Sydney zeigten neben einigen kommerziellen Sendern auch die BBC und das ZDF die mehrteilige Comedy-Sendung ,Road to Sydney“, die von Adidas finanziert und in Auftrag gegeben wurde. In je zwei- bis dreiminütigen Episoden trifft ein britischer Komiker auf Medaillenanwärter in diversen Disziplinen und fordert diese im Training heraus. Dies alles findet in einer komplett markenfreien Welt statt - bis auf die Adidas-Kleidung und Ausrüstung (Matthies 2004: 38f). Die Episoden 
wurden nicht als Werbespot oder einzelne Sendungen gezeigt, sondern als Element in einer redaktionellen Sportsendung („Grandstand“ auf BBC1) (Archer 2000). Aber nicht nur der Einfluss von Werbung treibende Unternehmen stellen den Public Value infrage. Im Sportkontext lässt sich auch eine Einflussnahme von Verbänden beobachten. Im Vorfeld der Olympischen Spiele in Beijing strahlten mehrere Public-Service-Sender Sendungen aus die statt des Gemeinwohls das Wohl des IOCs mehren sollten. Um die Ausstrahlungsrechte zu sichern, hatten sich die in der EBU zusammengeschlossenen Public-ServiceSender verpflichtet, PR-Spots des IOC kostenlos und als redaktionelles Programm getarnt auszustrahlen (Schraven 2008). Weiter mussten die EBU-Sender einen vom IOC in Auftrag gegebenen Film über Beijing ausstrahlen. Svergies Television sendete den Film zwar, distanzierte sich jedoch anschließend (Grimberg/Wolff 2008), die BBC verweigerte die Ausstrahlung. Eine unabhängige Berichterstattung dürfte so erschwert werden und die Unterhaltung wird zur Propaganda, die von der Kommerzialisierung des Sports ablenken soll. Die deutschen Public-Service-Sender zeigten sich nach den Spielen reumütig und wollen in Zukunft dafür sorgen, dass solche Klauseln „wegverhandelt“ werden (Grimberg 2008).

\section{Fazit}

Ähnlich wie in der Fernsehkritik mangels klarer Kriterien die Haltung „Ich erkenne Qualität, wenn ich sie sehe“ vorherrscht, könnte für die Bewertung von Public Value im Unterhaltungs-TV festgehalten werden: „Public Value merkt man, wenn er fehlt “. Selbst wenn Public Value als Qualität verstanden wird, die von der Gesellschaft zwar erwünscht, von den Individuen aber nicht genügend nachgefragt wird, lässt sich nicht einfach eine Checkliste aufstellen, die am Ende ein klares Ergebnis auswirft. Jenseits der unveräußerlichen Menschenrechte wird das gesellschaftlich erwünschte Gemeinwohl ständig neu ausgehandelt. Manche Aspekte mögen ganz verschwinden, andere in ihrer Priorität heraufoder herabgestuft werden. Prioritäten regeln die Verteilung von begrenzten Ressourcen. Die Bewertung des Public Value kann somit nicht völlig von den Kosten, die bei seiner Produktion entstehen, entkoppelt werden. Die Berücksichtigung von Minderheiteninteressen dient auch im Unterhaltungsbereich dem Public Value. Jedoch sollte die Frage erlaubt sein, welcher Ressourcenaufwand für wie viel Vielfalt von der Gesamtgesellschaft als gerechtfertigt erachtet wird. Und schließlich stellt sich die Frage, wie viel Public Value die Unterhaltung verträgt. Wir haben der Adidas-Serie „Road to Beijing“ einen Public Value abgesprochen, gleichwohl ist sie schreiend komisch. Schon 1970 stellte sich Alfred Biolek die Frage, ob man als Entertainer noch unterhaltend ist, wenn man alle Anforderungen des Public Value erfüllt: 
„Es ist nicht so schwierig zu erkennen, daß eine Unterhaltungssendung einem bestimmten Geschmack entsprechen muß, daß sie ein bestimmtes Niveau haben soll, daß sie realitätsbezogen oder gar zeitkritisch sein kann. Das Schwierige ist jedoch erst, auch bei Erfüllung all dieser Ansprüche immer noch eine Unterhaltungssendung zu machen" (zitiert in Prager 1971: 114).

\section{Literatur}

Albers, Robert (1992): Quality in television from the perspective of the professional program maker. In: Studies in Broadcasting. 28. 7-76

Albers, Robert (1996): Quality in programming from the perspective of the professional programme maker. In: Ishikawa (Hg.) (1996): 101-144

Amgarten, Gabriela (2008): Praxisperspektive: Unterhaltungsproduktion im Service public. In: Siegert/von Rimscha (Hg.) (2008): 116-122

Appel, Eva (Hg.) (2008): Ware oder Wert? Fernsehen zwischen Cash Cow und Public Value. Dokumentation der 41. Mainzer Tage der Fernsehkritik. Mainz: ZDF

Archer, Belinda (2000): Where's the logo? In: The Guardian vom 18.09.2000: 6

Binder, Susanne/Fartacek, Gebhard (2006a): Der Musikantenstadl als die unerforschte „Visitenkarte Österreichs“. In: Binder/Fartacek (Hg.): 14-23

Binder, Susanne/Fartacek, Gebhard (2006b): Der Musikantenstadl. Alpine Populärkultur im fremden Blick. Wien: Lit

Bleicher, Joan Kristin (2001). Mediengeschichte des Fernsehens. In: Schanze (Hg.): 490-518

Blumler, Jay G. (1991): In pursuit of programme range and quality. In: Studies of Broadcasting. 27. 191-206.

Blumler, Jay G./Nossiter, Thomas J. (Hg.) (1991): Broadcasting finance in transition. A Comparative Handbook. New York: Oxford University Press

Bosshart, Louis (2006): Theorien der Medienunterhaltung: Aus dem Nichts zur Fülle. In: Frizzoni (Hg.) (2006): 17-30

Burgelman, Jean-Claude/Pauwels, Caroline (1992): Audiovisual policy and cultural identity in small European states: The challenge of a unified market. In: Media, Culture \& Society. 14 (2). 169183

Costera Meijer, Irene (2005): Impact or content?: Ratings vs quality in public broadcasting. In: European Journal of Communication. 20 (1). 27-53

Dewenter, Ralf/Haucap, Justus (2009): Ökonomische Auswirkungen von öffentlich-rechtlichen Online-Angeboten. Marktauswirkungen innerhalb von Drei-Stufen-Tests: Gutachten im Auftrag des VPRT e.V. Online: http://www.vprt.de/get_asset_file.php?mid=19\&file=o_document_ 20090806151124_studie_2009_07_29_VPRT_HaucapDewenter_DST_Gutachten_Oekonomisc heAuswirkungen_Zusammenfassung.pdf (16.02.2010)

Donges, Patrick/Puppis, Manuel (Hg.) (2003): Die Zukunft des öffentlichen Rundfunks. Internationale Beiträge aus Wissenschaft und Praxis. Köln: Herbert von Halem

Erlei, Mathias (1992): Meritorische Güter: Die theoretische Konzeption und ihre Anwendung auf Rauschgifte als demeritorische Güter. Münster: LIT

Freedman, Des (2008): The politics of media policy. Oxford: Polity Press

Friedrichsen, Mike/Göttlich, Udo (Hg.) (2004): Diversifikation in der Unterhaltungsproduktion. Köln: Herbert von Halem

Frizzoni, Brigitte (Hg.) (2006): Unterhaltung. Konzepte, Formen, Wirkungen. Zürich: Chronos

Gehrau, Volker (2008): Fernsehbewertung und Fernsehhandlung: Ansätze und Daten zu Erhebung, Modellierung und Folgen von Qualitätsurteilen des Publikums über Fernsehangebote. München: Reinhard Fischer 
Gottschalk, Hans (1971): Die politische Wirksamkeit der unpolitischen Unterhaltung. In: Prager (Hg.) (1971): 103-111

Grimberg, Steffen (2008): Ade, Fahnenschwenker! In: die tageszeitung vom 30.08.2008: 15.

Grimberg, Steffen/Wolff, Reinhard (2008): Das IOC lässt senden: Kostenlose Werbung für Olympia. In: die tageszeitung vom 05.08.2008: 18

Hall, Peter Christian (Hg.) (1995): Qualität hat ihren Preis. Die Zukunftssicherung des öffentlichrechtlichen Fernsehens. Mainz: ZDF

Heygster, Anna-Luise/Stolte, Dieter (Hg.) (1980): Wirklichkeit und Fiktion im Fernsehspiel. Mainz: v. Hase und Koehler

Hoffmann-Riem, Wolfgang (2003): Kann und soll der öffentliche Rundfunk eine Staatsaufgabe sein? In: Donges/Puppis (Hg.) (2003): 29-51

Holznagel, Bernd/Vollmeier, Ines (2003): Gemeinsame oder getrennte Aufsichten? Ein Überblick über die verschiedenen Ansätze der Beaufsichtigung von öffentlichem und kommerziellem Rundfunk. In: Donges/Puppis (Hg.) (2003): 277-291

Hömberg, Walter/Pürer, Heinz (Hg.) (1996): Medien-Transformation. Zehn Jahre dualer Rundfunk in Deutschland. Konstanz: UVK

Ishikawa, Sakae (Hg.) (1996): Quality assessment of television, Luton: University of Luton Press

Janke, Hans (1980): Interesse und Vermittlung: Was Kritik kritisiert. In: Heygster/Stolte (Hg.) (1980): 71-82

Jarren, Otfried (1996): Das duale Rundfunksystem - politiktheoretisch betrachtet. In: Hömberg/ Pürer (Hg.) (1996): 69-80

Kissler, Alexander (2009): Dummgeglotzt: Wie das Fernsehen uns verblödet. Gütersloh: Gütersloher Verlagshaus

Klär, Karl-Heinz (1995): Qualität hat nicht nur einen geldlichen Gegenwert: Man muß sich ihr persönlich verschreiben. In: Hall (Hg.) (1995): 265-267

Klingler, Walter/Roters, Gunnar/Zöllner, Oliver (Hg.) (1998): Fernsehforschung in Deutschland. Themen, Akteure, Methoden. Baden-Baden: Nomos

Konzession SRG: Konzession für die SRG SSR idée suisse vom 28. November 2007

Leggatt, Timothy (1993): Quality in television: The views of professionals. In: Studies of Broadcasting. 29. 37-69

Lobigs, Frank (2004): Niveauvolle Unterhaltung im öffentlich-rechtlichen Fernsehen: Notwendige Voraussetzungen eines Arguments der Meritorik. In: Friedrichsen/Göttlich (Hg.) (2004): 48-64

Matthies, Anja (2004): Virtuelle Werbung: Rechtliche Fragen bei der Übertragung von Sportereignissen im Fernsehen. Hamburg: Kovač

Metz, Markus/Seeßlen, Georg (2009): Von Tutti Frutti ins Dschungelcamp: Privatfernsehen in Deutschland, Zündfunk auf Bayern 2 Radio am 18.10.2009.

Niggemeier, Stefan (2008): Public Value ist, wenn ... In: Appel (Hg.) (2008): 155-161

Nossiter, Thomas J. (1991): British television: A mixed economy. In: Blumler/Nossiter (Hg). (1991): 95-143

ORF-G: Bundesgesetz über den Österreichischen Rundfunk vom 21. September 1984 (zuletzt geändert am 28.12.2007)

Prager, Gerhard (Hg.) (1971): Unterhaltung und Unterhaltendes im Fernsehen. Mainz: v. Hase und Koehler

Puppis, Manuel (2009): Media regulation in small states. In: International Communication Gazette. 71 (7). $7-17$

RTVG: Bundesgesetz vom 24. März 2006 über Radio und Fernsehen (RTVG). SR-Nummer: 784.40

RTVV: Radio- und Fernsehverordnung vom 9. März 2007 (RTVV). SR-Nummer: 784.401

Schanze, Helmut (Hg.) (2001): Handbuch der Mediengeschichte. Stuttgart: Alfred Kröner.

Schatz, Heribert/Schulz, Winfried (1992): Qualität von Fernsehprogrammen: Kriterien und Methoden zur Beurteilung von Programmqualität im dualen Fernsehsystem. In: Media Perspektiven. 11. 690-712 
Scheuch, Erwin K. (1971): Unterhaltung als Pausenfüller: Von der Vielfalt der Unterhaltungsfunktionen in der modernen Gesellschaft. In: Prager (Hg.) (1971): 13-46

Schlote, Axel/Latzel, Peter (1998): Bewertung von Fernsehsendungen: Chancen und Probleme einer Objektivierung von Programmentscheidungen. In: Klingler et al. (Hg.) (1998): 815-837

Schraven, David (2008): Skurrile TV-Verträge von ARD und ZDF. In: Die Welt vom 23.08.2008: 32

Shusterman, Richard (2003): Entertainment: A question for aesthetics. In: British Journal of Aesthetics. 43 (3). 289-307

Siegert, Gabriele/von Rimscha, Bjørn (Hg.) (2008): Zur Ökonomie der Unterhaltungsproduktion, Köln: Herbert von Halem

Stumm, Mascha-Maria/Dorsch-Jungsberger, Petra E. (1996): Unterhaltungstheoreme bei Platon und Aristoteles: Eine Rückkehr zu den Ursprüngen der Diskussion um Funktionen und Wirkungen von Unterhaltung und der Versuch einer Auswertung fachfremder Literatur als Beitrag zur Klärung des kommunikationswissenschaftlichen Unterhaltungsbegriffes. Berlin: Vistas

Wildberger, Jürg (1994): Das Instrument der Sendererfolgskontrolle.: Schweizer Fernsehen DRS: Mehr Zuschauernähe und schärferes Programmprofil. In: Media Perspektiven. 2. 63-66

Wober, J. Mallory (1990): The assessment of television quality: Some explorations of methods and their results: IBA Research Department research paper. London 